\title{
Field Theory Design of Ferrite-Loaded Waveguide Nonreciprocal Phase Shifters with Multisection Ferrite or Dielectric Slab Impedance Transformers
}

\author{
JAROSLAW UHER, FRITZ ARNDT, SENIOR MEMBER, IEEE, AND JENS BORNEMANN
}

\begin{abstract}
Impedance-matched ferrite-loaded waveguide nonreciprocal phase shifters are designed using the method of field expansion into eigenmodes, which includes higher order mode interaction between the step discontinuities. Computer-optimized $K u$-band ferrite stepped design examples, of $45^{\circ}$ and $90^{\circ}$ nonreciprocal differential phase shifts, attain typically about $2^{\circ}$ phase error and less than $-25 \mathrm{~dB}$ input reflection within a bandwidth of about 5 percent. Compact designs are achieved by thicker uniform ferrite slabs with dielectric transformer sections at each end. The theory is verified by comparison with available results from measurements and other methods.
\end{abstract}

\section{INTRODUCTION}

$\mathrm{N}$ ONRECIPROCAL PHASE SHIFT may be realized in rectangular waveguide by placing a ferrite slab [1]-[20] in a plane (Fig. 1) where the magnetic field is nearly circularly polarized [2]-[25]. The slab is biased transversely by a dc magnetic field sufficiently below that at which ferromagnetic resonance occurs. Whereas for a similar reciprocal structure-a dielectric-slab-loaded phase shifter-solutions for the impedance matching to the unloaded waveguide sections are well known [26], [27], [32], the three-dimensional field theory problem of related stepped anisotropic ferrite slab structures of finite length asymmetrically placed in the waveguide (Fig. 1) has not yet been attacked sufficiently. Suitably optimized structures, however, may be of considerable importance for many applications, e.g., for differential phase shift circulators [28], where good overall performance depends on the requirement that the individual components have appropriate electrical characteristics. Moreover, appropriate impedance transformer sections allow compact nonreciprocal phase shifter designs, since thick slab structures may then be utilized which otherwise would cause VSWR values which are unacceptably high.

Many investigations concerning ferrite-loaded waveguides have been reported [3], [4], [7], [9]-[16], [18]-[25], [29]-[31], [34], including analyses of discontinuity prob-

Manuscript received July 17, 1986; revised December 29, 1986. This work was supported in part by the German Research Society (DFG) under Contract Ar 138/6-1.

The authors are with the Microwave Department, University of Bremen, D-2800 Bremen, West Germany.

IEEE Log Number 8714120.

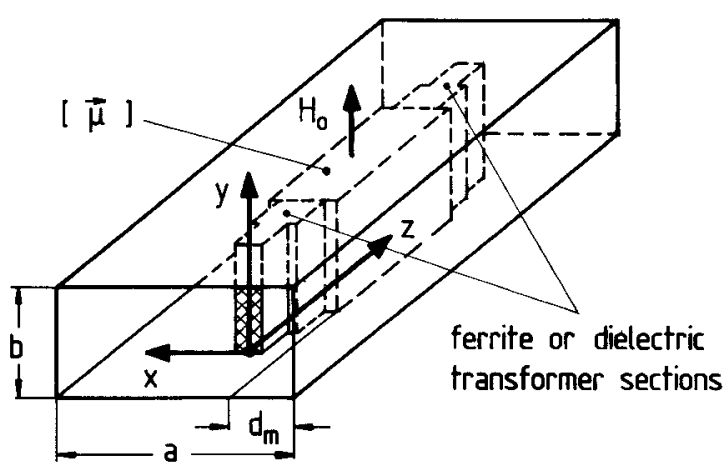

(a)

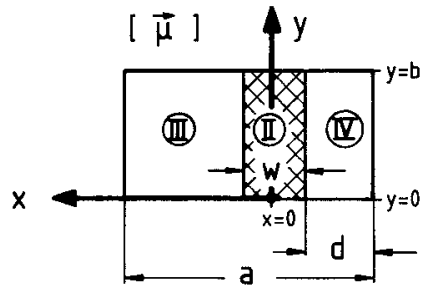

(b)

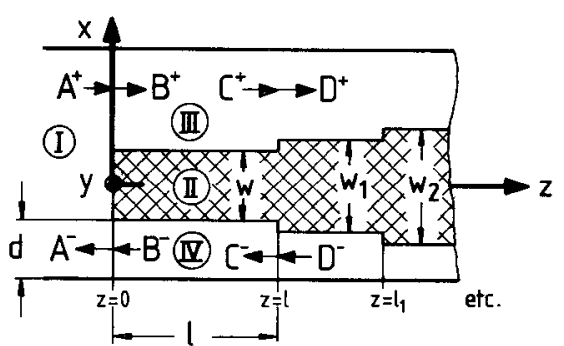

(c)

Fig. 1. Ferrite-loaded waveguide nonreciprocal phase shifter with multisection ferrite or dielectric slab impedance transformers at both ends. (a) Waveguide with the slab insert. (b) Cross section of the slab structure. (c) Wave amplitude vectors of the structures of finite length.

lems by a coupled-line model [24] and by modal expansion methods [18]-[20], [23]. These analyses, however, are limited to uniform ferrite slab structures [18], [20], [23], [24] or a structure periodically loaded by an array of diaphragms [19]. Waveguide $H$-plane junctions with a ferrite post have been analyzed by an integral equation 
method [29], the modal expansion method [30], and the finite-element method [34]. However, these investigations are concerned with the performance of Y-junction circulators; consequently, ferrite posts with smooth boundaries such as a circular and a triangular post, with and without depressed sides, are studied [29], [30], [34].

This paper describes a modal-S-matrix method for designing ferrite-slab-loaded nonreciprocal waveguide phase shifters with multisection impedance transformers (Fig. 1) at both ports. The transformer sections may be formed of the same ferrite material as that used for the middle section of the phase shifter, or may advantageously consist of appropriate dielectric material which can often be handled better mechanically. Moreover, dielectric impedance transformer sections fastened to the uniform ferrite slab require no addition dc magnetic field. Since good VSWR characteristics can be realized, relatively thick ferrite-slab phase-shifting structures may be included in the optimization procedure, which, consequently, leads to compact designs. The method of field expansion into incident and scattered waves at all discontinuities includes the higher order mode coupling effects along the whole three-dimensional structure. Suitably optimized geometric parameters improye the nonreciprocal phase characteristic; dielectric losses are also considered in the calculations. The computer-aided design using this theory provides low-input VSWR behavior together with a prescribed differential nonreciprocal phase shift. The theory is verified through comparison with computed and measured results available from the literature as well as with our own measurements.

\section{THEORY}

For each homogeneous subregion, $\nu=\mathrm{I}$ to IV (Fig. 1(b) and (c)), the fields [7] of the resulting $\mathrm{TE}_{n 0}$ waves, if a $\mathrm{TE}_{m 0}$ wave is incident,

$$
\begin{array}{ll}
\nabla \times \vec{H}=j \omega \epsilon \vec{E} & \nabla \cdot(\langle\vec{\mu}\rangle \vec{H})=0 \\
\nabla \times \vec{E}=-j \omega\langle\vec{\mu}\rangle \vec{H} & \nabla \cdot \vec{E}=0
\end{array}
$$

are derived from the electric field component $\vec{e}_{y} E_{y}^{(v)}$. This component may be expressed as a sum of $N$ eigenmodes [3], [23] satisfying the vector Helmholtz equation and the boundary condition at $x=-d-w / 2$, or $x=a-d-$ $w / 2$, respectively:

$$
E_{y}^{(\nu)}=\sum_{n=1}^{N}\left\{\begin{array}{l}
E_{n}^{(\mathrm{I})} \sin \frac{n \pi}{a}\left(x+d+\frac{w}{2}\right) \\
E_{n}^{(\mathrm{IV})} \sin k_{x n}^{(\mathrm{IV})}\left(x+d+\frac{w}{2}\right) \\
E_{n}^{(\mathrm{II})} \sin k_{x n}^{(\mathrm{II})}(x)+F_{n}^{(\mathrm{II})} \cos k_{x n}^{(\mathrm{II})}(x) \\
E_{n}^{(\mathrm{III})} \sin k_{x n}^{(\mathrm{III})}\left(a-d-\frac{w}{2}-x\right)
\end{array}\right.
$$

where a $z$ dependence of $\exp \left(-\gamma_{n} z\right)$ is understood. For a dc magnetic field $H_{0}$ in the $y$ direction, the permeability tensor takes the form [1]--[6]

$$
\langle\vec{\mu}\rangle=\mu_{0}\left(\begin{array}{ccc}
\mu_{1} & 0 & -j \kappa \\
0 & \mu_{r} & 0 \\
j \kappa & 0 & \mu_{1}
\end{array}\right)
$$

with elements $\mu_{1}, \mu_{r}$, and $\kappa$ given in [1]-[3]. The relations for the wavenumbers in (2) are

$$
\begin{array}{ll}
k_{0}^{2}=\omega^{2} \mu_{0} \epsilon_{0} & k_{0}^{2}=\left(k_{x n}^{(\mathrm{III}),(\mathrm{IV})}\right)^{2}-\gamma_{n}^{2} \\
\mu_{2}=\frac{\mu_{1}^{2}-\kappa^{2}}{\mu_{1}} & k_{0}^{2} \epsilon_{r} \mu_{2}=\left(k_{x n}^{(\mathrm{II})}\right)^{2}-\gamma_{n}^{2}
\end{array}
$$

where $\gamma_{n}$ is determined via field matching [7] of the transverse field components along the boundaries $x=$ $\pm w / 2$. The requirement that the system determinant be zero results in a transcendental equation for $\gamma_{n}$, which is solved numerically. The Newton method has turned out to yield numerically stable solutions for thick slabs provided that the starting values are taken from known thin-ferriteslab expressions [31], [23] and that the step intervals for increasing thickness are chosen to be sufficiently small.

For calculation of the modal scattering matrix of the three-dimensional structure (Fig. 1(c)), the related biorthogonality relations [7], [9], [11], [23] for anisotropic structures have to be taken into account:

$$
\left(\gamma_{n}+\gamma_{m}^{\prime}\right) \iint_{F}\left[\left(\vec{E}_{n} \times \vec{H}_{m}^{\prime}-\vec{E}_{m}^{\prime} \times \vec{H}_{n}\right) \cdot \vec{e}_{z}\right] d F=0 .
$$

Here, the reciprocity principle involves the fields $E_{m}^{\prime}, H_{m}^{\prime}$, and $\gamma_{m}^{\prime}$ which are solutions to the related problem with media characterized by the transposed permeability tensor [7]. Matching of the transversal field components (1), (2) at the corresponding interfaces (Fig. 1(c)) at $z=0$ yields

$$
\begin{aligned}
\sum_{m=1}^{M}\left(A_{m}^{+}+A_{m}^{-}\right) T_{m} u_{m} & =\sum_{n=1}^{N}\left(B_{n}^{+} e_{n}^{\mathrm{F}}+B_{n}^{-} e_{n}^{\mathrm{R}}\right) \\
\sum_{m=1}^{M}\left(A_{m}^{+}-A_{m}^{-}\right) T_{m} u_{m} Y_{m} & =\sum_{n=1}^{N}\left(B_{n}^{+} h_{n}^{\mathrm{F}}-B_{n}^{-} h_{n}^{\mathrm{R}}\right)
\end{aligned}
$$

where

$$
\begin{aligned}
u_{m} & =\sin \left(\frac{\pi m}{a}\left(x+d+\frac{w}{2}\right)\right), \\
Y_{m} & =\frac{k_{z m}}{\omega \mu_{0}}, \\
k_{z m}^{2} & =k_{0}^{2}-\left(\frac{m \pi}{a}\right)^{2},
\end{aligned}
$$

$T_{m} \quad$ normalization factor so that the power carried by a given wave is $1 \mathrm{~W}$ for a wave amplitude of $1 \sqrt{W}$ [7],

$e_{n}^{\mathrm{F}, \mathrm{R}} \quad$ normalized eigenfunction of the $E_{y}$ component of the forward $(F)$ or reverse $(\mathrm{R}) n$th mode in region $0<z<l$, respectively,

$h_{n}^{\mathrm{F}, \mathrm{R}}$ normalized eigenfunction of the $H_{x}$ component of the forward $(\mathrm{F})$ or reverse $(\mathrm{R}) n$th mode in region $0<z<l$, respectively.

The still unknown amplitude coefficients $A_{m}^{+}, A_{m}^{-}, B_{n}^{+}$, and $B_{n}^{-}$in (6) can be related to each other through the 


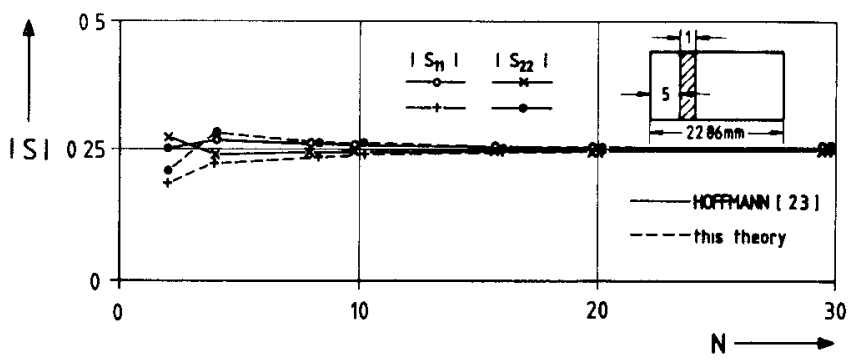

Fig. 2. Magnitude of the reflection coefficients $S_{11}$ and $S_{22}$ of a ferrite slab as a function of the number $N$ of eigenmodes considered. Slab length $l=60 \mathrm{~mm} ; f=9.36 \mathrm{GHz} ; \mu=0.4-j 0.06, \kappa=-1 .-j 0.06$; $\epsilon_{r}=12.5-j 0.005$.

modal scattering matrix at the step discontinuity at $z=0$ :

$$
\left(\begin{array}{l}
(A)^{-} \\
(B)^{+}
\end{array}\right)=\left(\begin{array}{ll}
\left(S_{11}\right)^{0} & \left(S_{12}\right)^{0} \\
\left(S_{21}\right)^{0} & \left(S_{22}\right)^{0}
\end{array}\right)\left(\begin{array}{l}
(A)^{+} \\
(B)^{-}
\end{array}\right)
$$

where the coefficients are given in the Appendix.

The general modal scattering matrix of a ferrite-slabloaded waveguide structure of finite length $l$ is given by

$$
\left(\begin{array}{c}
(A)^{-} \\
(D)^{+}
\end{array}\right)=\left(\begin{array}{ll}
\left(S_{11}\right)^{g} & \left(S_{12}\right)^{g} \\
\left(S_{21}\right)^{g} & \left(S_{22}\right)^{g}
\end{array}\right)\left(\begin{array}{c}
(A)^{+} \\
(D)^{-}
\end{array}\right)
$$

where these coefficients are also given in the Appendix. The overall scattering matrix of two and more sections (Fig. 1(c)) is calculated by directly combining the appropriate single scattering matrices as shown explicitly in [32]. For computer optimization [27] of the transformer section lengths, the expansion into ten eigenmodes at each discontinuity has turned out to be sufficient. The final design data are proven through an expansion of 30 eigenmodes.

The convergence behavior of the modal expansion method used is demonstrated in Fig. 2 by showing the magnitude of the reflection coefficients $S_{11}$ and $S_{22}$ for a single ferrite slab as a function of the number $N$ of eigenmodes considered in (2). Sufficient coincidence between $\left|S_{11}\right|$ and $\left|S_{22}\right|$ (according to the reciprocity relationships for gyrotropic media) and good agreement with Hoffmann's results [23] are obtained already for $N=10$.

\section{RESULTS}

In Fig. 3, the magnitude of the reflection coefficient for a lossy dielectric obstacle of finite length in a R100 waveguide $(22.860 \mathrm{~mm} \times 10.160 \mathrm{~mm})$ is shown as a function of frequency. The calculated results agree well with the theoretical and measured values of Chaloupka [24].

For a uniform ferrite slab of finite length, frequencydependent results were published by Gardiol et al. [14] and Hoffmann et al. [33]. For comparison, computations were carried out for these geometries and materials. The results are presented in Fig. 4, showing good agreement between our theory and the experimental and theoretical values of [14] and [33]. For a slab of polycrystalline YIG on a

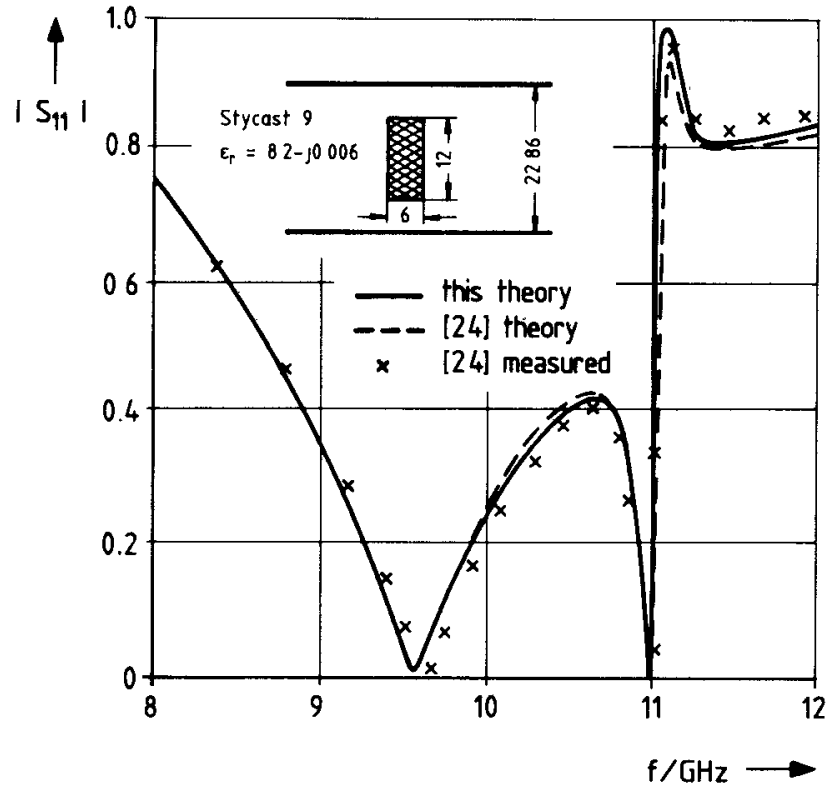

Fig. 3. Lossy dielectric obstacle of finite length in a R100 waveguide $(22.860 \mathrm{~mm} \times 10.160 \mathrm{~mm})$. Magnitude of the input reflection coefficient as a function of frequency. Comparison with [24].

narrow wall, which can be used for tunable filters [14], the (higher) attenuation in the reverse direction of propagation is plotted versus frequency (Fig. 4(a)). The internal magnetic field was chosen as in [14]. For an R1 ferrite slab structure of length $l=74.9 \mathrm{~mm}$ within a R100 waveguide housing $(22.860 \mathrm{~mm} \times 10.160 \mathrm{~mm})$, the propagation factors (Fig. 4(b)) calculated from the related eigenvalue problem are compared with the measured results of [33] as a function of frequency. The amplitudes and phases of the forward $\left(S_{21}\right)$ and backward $\left(S_{12}\right)$ transmission coefficients are presented in parts (c) and (d) of Fig. 4 and compared with measured results from [33].

Fig. 5 shows the comparison of the theory with our own measurements (Fig. 5(a) for a ferrite TTI-2800 slab and Fig. 5(b) for a ferrite TTVG-1200 slab) within an R140 waveguide housing $(15.799 \mathrm{~mm} \times 7.899 \mathrm{~mm})$. Because of the relatively small gap width of the biasing magnet available for the experiment, only relatively short slabs could be chosen; for small slab widths, this results in a relatively low forward-to-backward nonreciprocal differential phase shift $\left(\Delta \phi=\operatorname{arc}\left(S_{21}\right)-\operatorname{arc}\left(S_{12}\right)\right)$. In Fig. 5(a), at $f=16.8 \mathrm{GHz}$, the higher order $\mathrm{TE}_{20}$ mode cutoff frequency, there is a rapid phase change of $180^{\circ}$, and the return loss increases. For different separations $d$ of the slab from the waveguide wall, increasing or decreasing values for $\Delta \phi$ as a function of frequency may be observed (Fig. 5(b)). A suitably chosen parameter $d$, therefore, may be utilized to improve the bandwidth of the phase characteristic (cf. also Fig. 6). As may be observed from Fig. 5, our theory is found to be in good agreement with the measured results.

For the measurements, an HP 8410 network analyzer was used. The ferrite slab probes have been fabricated by a grinding process with a deviation from the designed values 


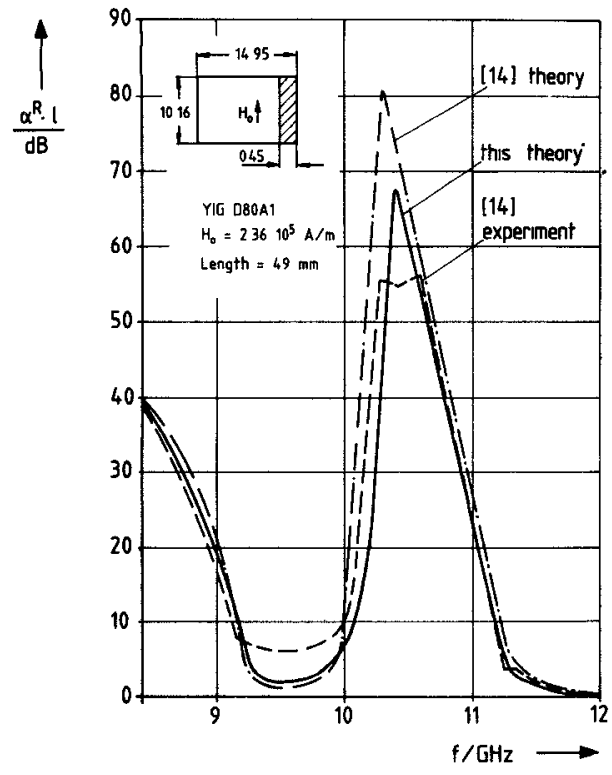

(a)

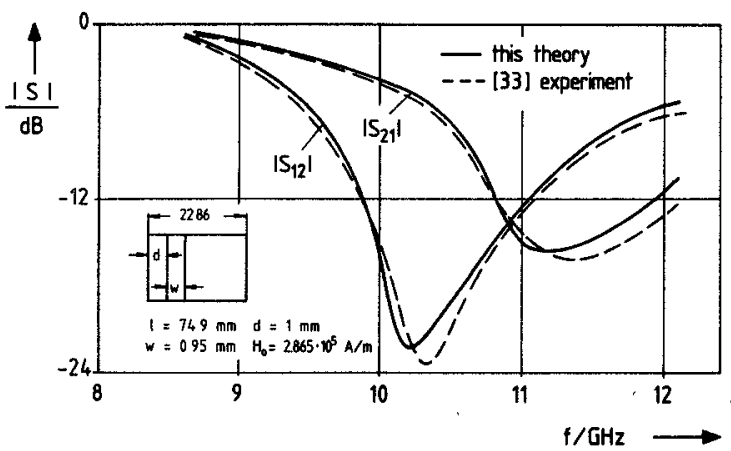

(c)

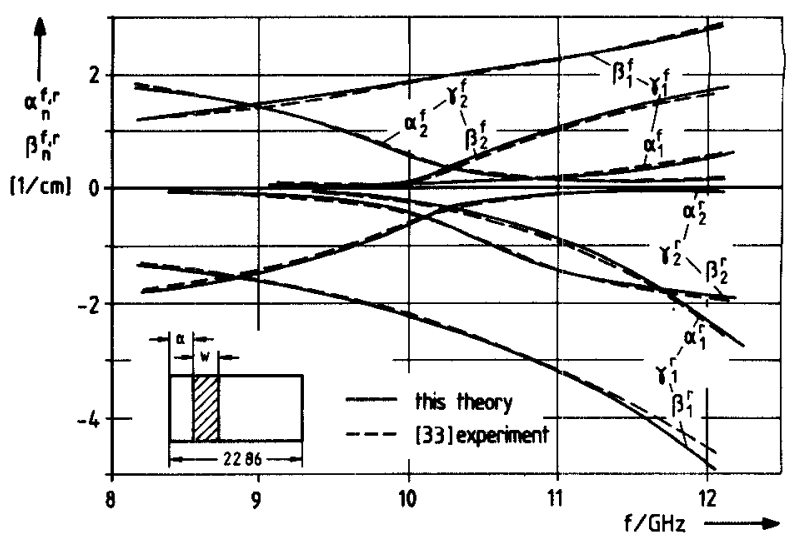

(b)

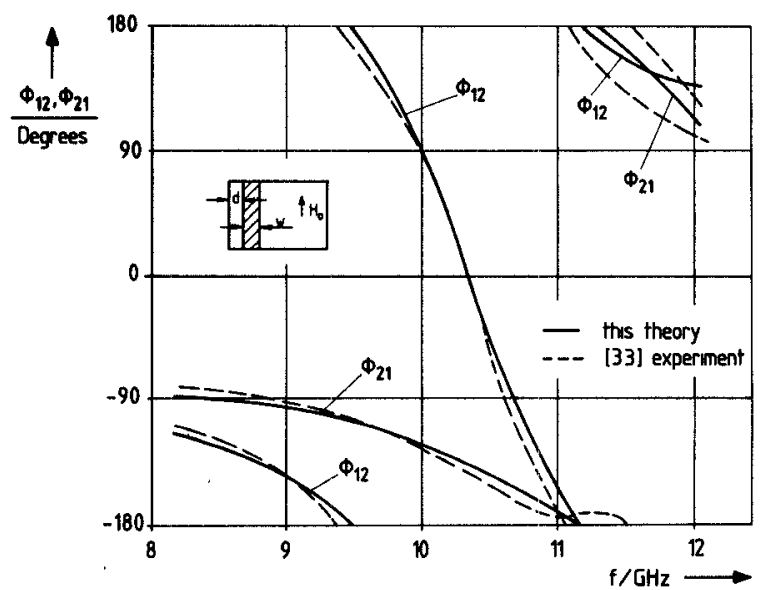

(d)

Fig. 4. Uniform ferrite slab of finite length in waveguide. (a) Attenuation in the reverse direction of a slab of polycrystalline YIG as a function of frequency. Comparison with [14]. (b) Propagation factors in forward (f) and reverse (r) direction calculated for a ferrite $\mathrm{R} 1$ slab structure of finite length $l=74.9 \mathrm{~mm}$ within a R100 waveguide. Comparison with experimental results from [33]. (c) Amplitudes and (d) phases of the forward $S_{21}$ and backward $S_{12}$ transmission coefficients for a ferrite $\mathrm{R} 1 \mathrm{slab}$ structure of $l=74.9 \mathrm{~mm}$ within a R100 waveguide. Comparison with experimental results from [33].

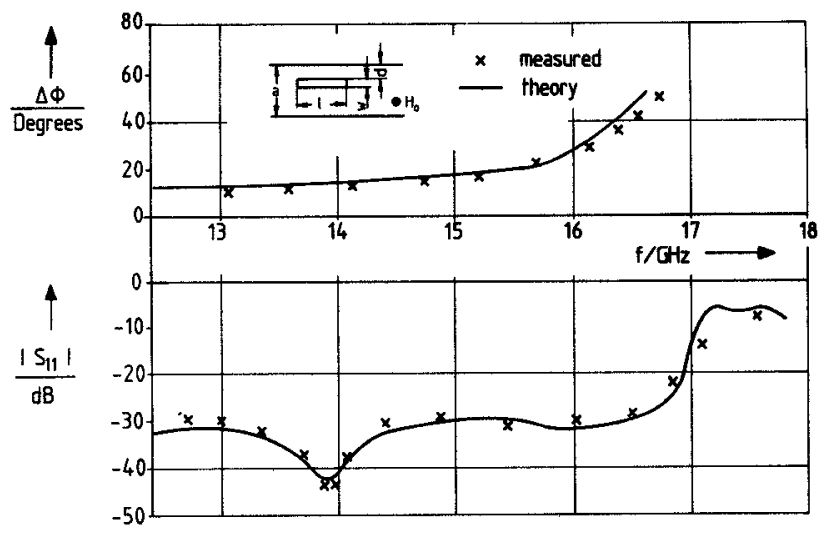

(a)

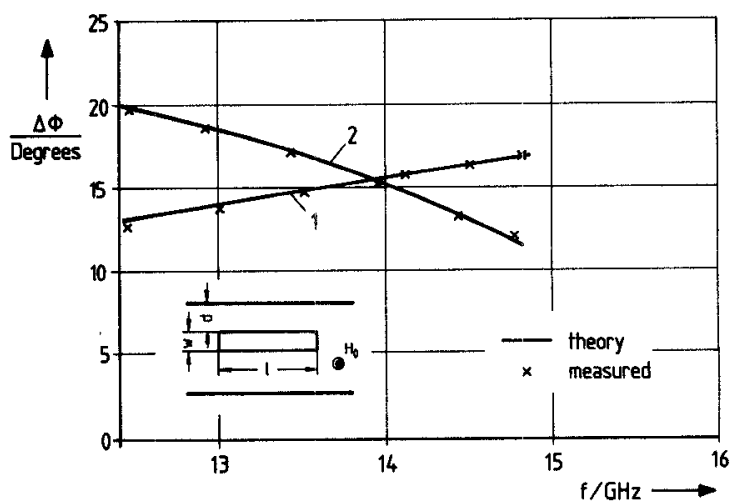

(b)

Fig. 5. Uniform ferrite slab of finite length in waveguide. (a) Nonreciprocal differential phase shift $\Delta \varphi=\varphi_{21}-\varphi_{12}$ and input reflection coefficient in decibels as a function of frequency. Ferrite TTI-2800 slab within a R140 waveguide (15.799 $\mathrm{mm} \times 7.898 \mathrm{~mm}), l=20 \mathrm{~mm}, d=0.7 \mathrm{~mm}, w=0.7 \mathrm{~mm}, H_{0}=1.2 \cdot 10^{5} \mathrm{~A} / \mathrm{m}$. Comparison with measurements (x). (b) Nonreciprocal differential phase shift $\Delta \varphi$ as a function of frequency with displacement $d$ as a parameter. Curve $1: d=1$ $\mathrm{mm}$; curve 2: $d=2 \mathrm{~mm}$. Ferrite TTVG-1200 slab within a R140 waveguide, $l=37.5 \mathrm{~mm}, w=0.5 \mathrm{~mm}, H_{0}=1.2 \cdot 10^{5} \mathrm{~A} / \mathrm{m}$. Comparison with measurements (x). 


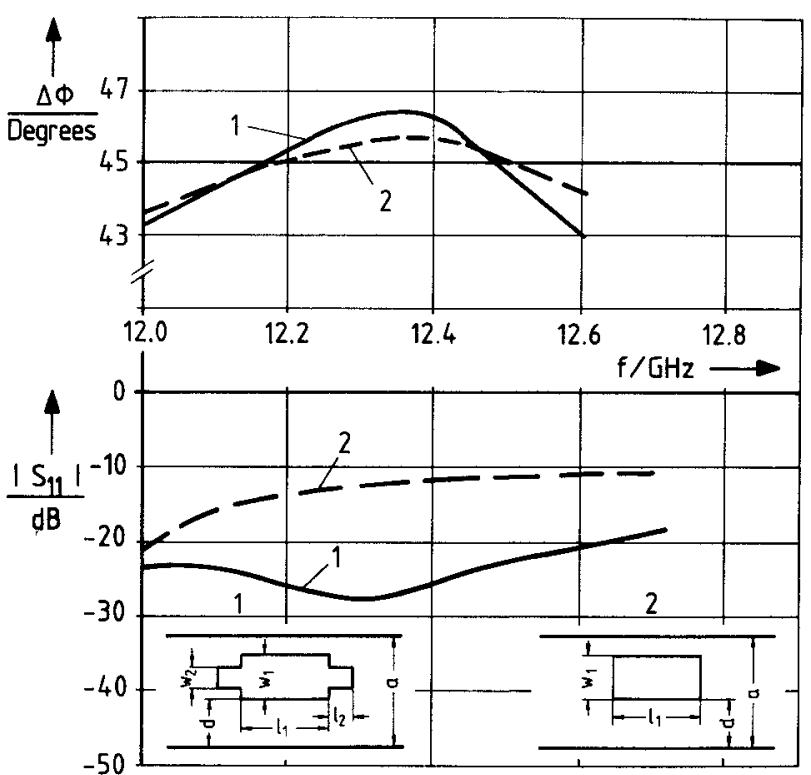

(a)

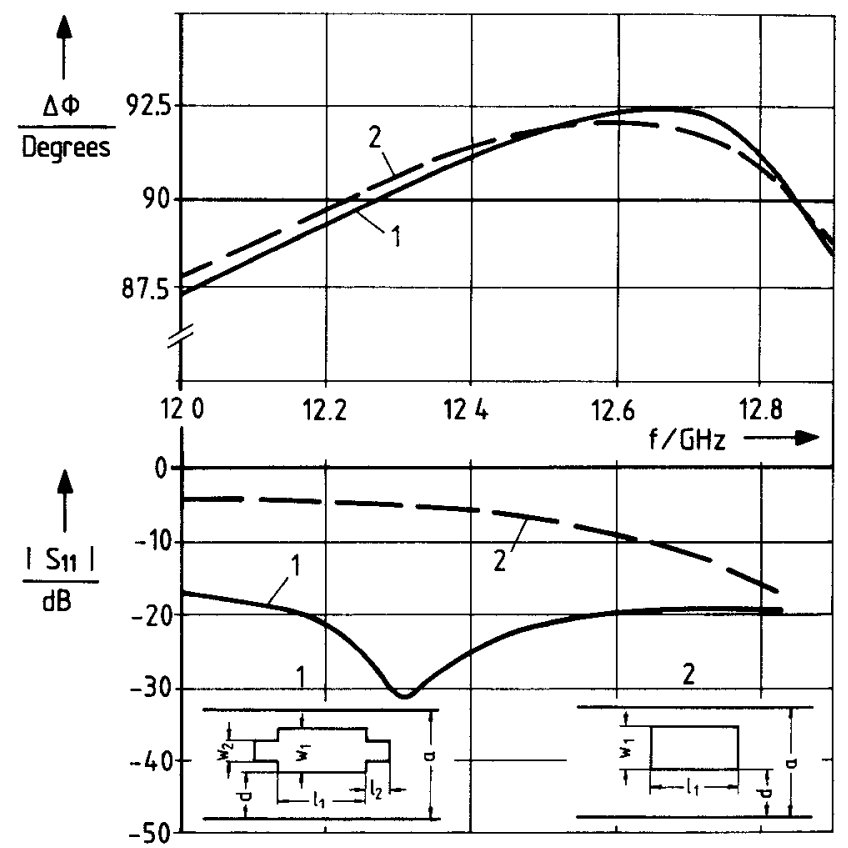

(b)

Fig. 6. Optimized nonreciprocal phase shifters with one-step ferrite impedance transformer sections. Nonreciprocal differential phase shift and input reflection coefficient in decibels as a function of frequency. Ferrite TTVG-1200 slab within R140 waveguide. Curve 1: with impedance transformers at both ends; curve 2: without impedance transformers. (a) Design for $\Delta \varphi=45^{\circ} \pm 2^{\circ}$. Curve 1: $w_{1}=1.2 \mathrm{~mm}, w_{2}=0.6$ $\mathrm{mm}, l_{1}=13.2 \mathrm{~mm}, l_{2}=7.3 \mathrm{~mm}, d=13.55 \mathrm{~mm}, H_{0}=1.2 \cdot 10^{5} \mathrm{~A} / \mathrm{m}$. Curve 2: $w_{1}=1.2 \mathrm{~mm}, l_{1}=14 \mathrm{~mm}, d=13.55 \mathrm{~mm}, H_{0}=1.2 \cdot 10^{5} \mathrm{~A} / \mathrm{m}$. (b) Design for $\Delta \varphi=90^{\circ} \pm 2.5^{\circ}$. Curve 1: $w_{1}=1.2 \mathrm{~mm}, w_{2}=0.7 \mathrm{~mm}$, $l_{1}=21 \mathrm{~mm}, l_{2}=7.8 \mathrm{~mm}, d=13.55 \mathrm{~mm}, H_{0}=1.2 \cdot 10^{5} \mathrm{~A} / \mathrm{m}$. Curve $2:$ $w_{1}=1.2 \mathrm{~mm}, l_{1}=28 \mathrm{~mm}, d=13.55 \mathrm{~mm}, H_{0}=1.2 \cdot 10^{5} \mathrm{~A} / \mathrm{m}$.

of about 1-2 percent. The lateral displacement within the R140 waveguide housing was determined by appropriate measuring gauges. An electromagnet fixed at the waveguide housing yields the biasing dc magnetic field, which was measured by a gaussmeter. The internal magnetic field was calculated according to the procedure given in [35].

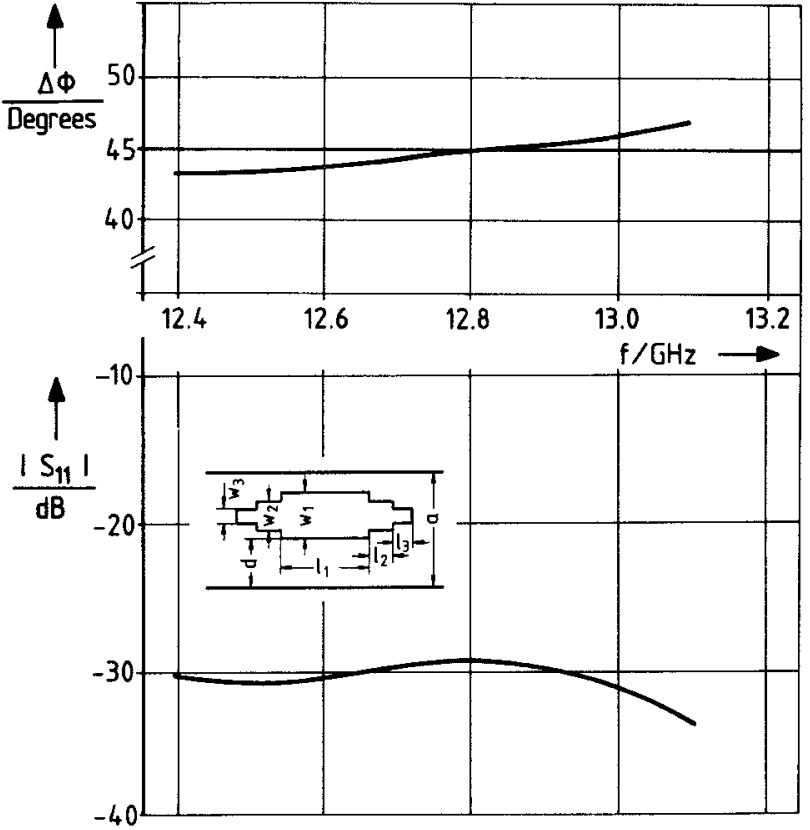

(a)

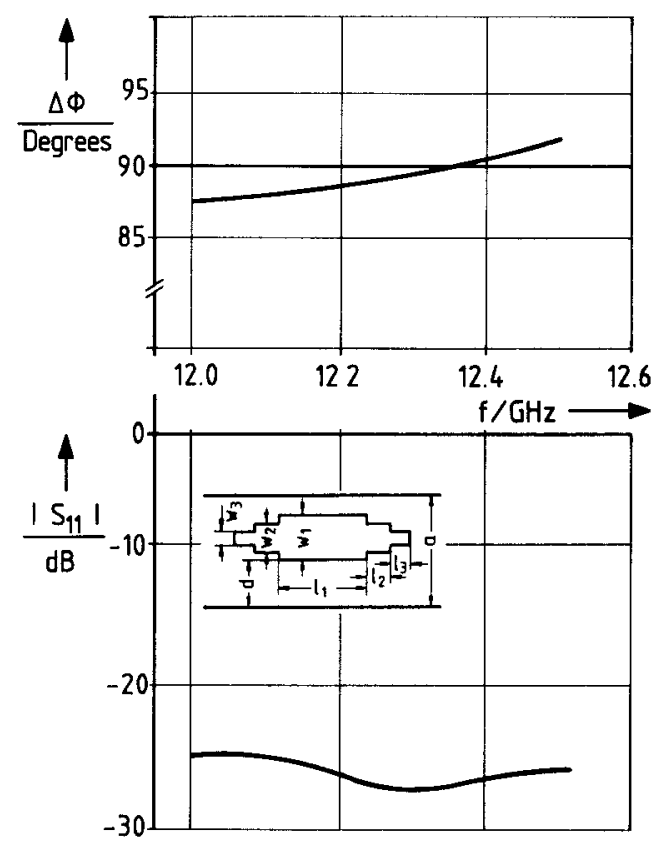

(b)

Fig. 7. Optimized nonreciprocal phase shifters with two-step ferrite impedance transformer sections. Nonreciprocal differential phase shift and input reflection coefficient in decibels as a function of frequency. Ferrite TTVG-1200 slab within R140 waveguide. (a) Design for $\Delta \varphi=$ $45^{\circ} \pm 1.7^{\circ}: w_{1}=0.7 \mathrm{~mm}, w_{2}=0.5 \mathrm{~mm}, w_{3}=0.3 \mathrm{~mm}, l_{1}=100 \mathrm{~mm}$, $l_{2}=9 \mathrm{~mm}, l_{3}=10 \mathrm{~mm}, d=14.4 \mathrm{~mm}, H_{0}=1.2 \cdot 10^{5} \mathrm{~A} / \mathrm{m}$. (b) Design for $\Delta \varphi=90^{\circ} \pm 2.5^{\circ}: w_{1}=0.8 \mathrm{~mm}, w_{2}=0.6 \mathrm{~mm}, w_{3}=0.4 \mathrm{~mm}, l_{1}=130$ $\mathrm{mm}, l_{2}=9.5 \mathrm{~mm}, l_{3}=10.5 \mathrm{~mm}, d=14.25 \mathrm{~mm}, H_{0}=1.2 \cdot 10^{5} \mathrm{~A} / \mathrm{m}$.

Design examples for optimized nonreciprocal phase shifters with one-step ferrite impedance transformer sections are presented in Fig. 6. To verify the improvement of the VSWR behavior by additional impedance transformer sections (curves 1), the corresponding uniform slab phase shifter behavior is included in the presentation (curves 2). 


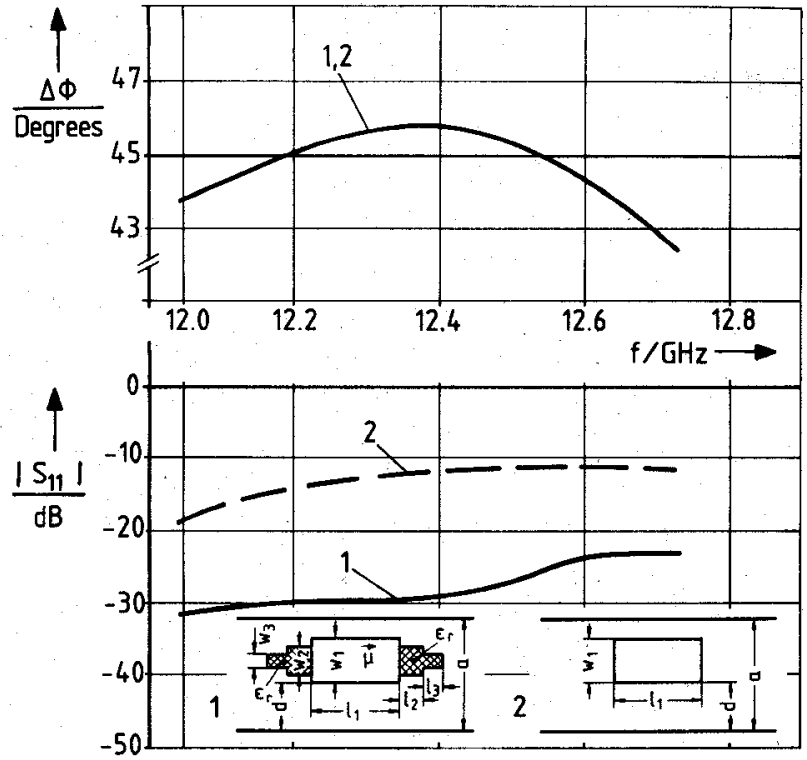

(a)

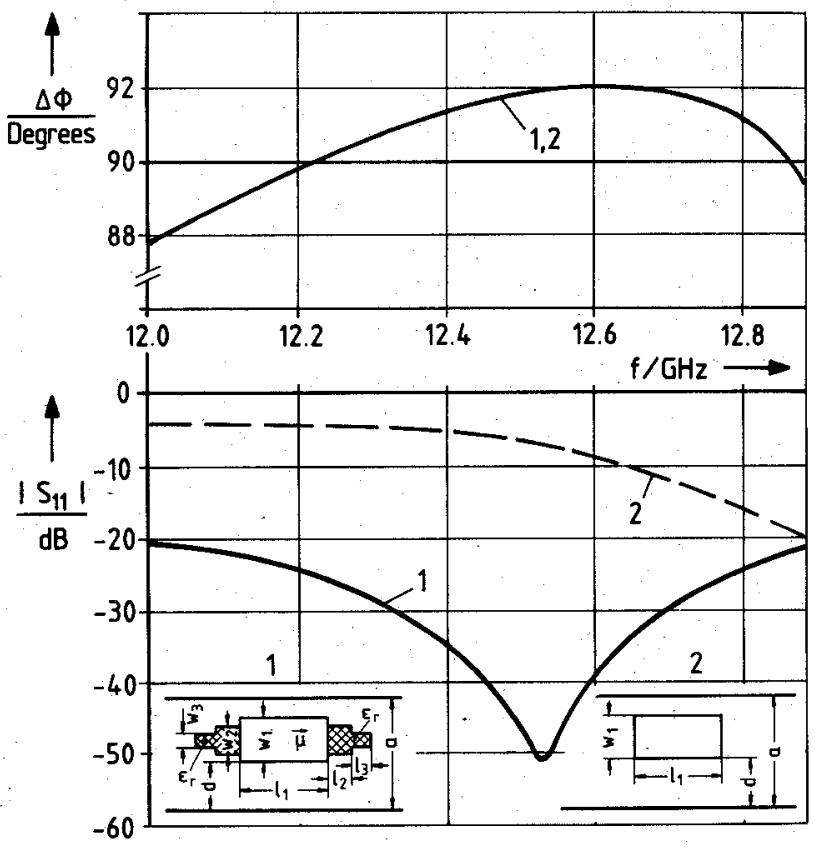

(b)

Fig. 8. Optimized compact nonreciprocal phase shifters with two-step dielectric impedance transformer sections. Nonreciprocal differential phase shift and input reflection coefficient in decibels as a function of frequency. Ferrite TTVG-1200 slab within R140 waveguide. Curve 1: with impedance transformers at both ends; curve 2: without impedance transformers. (a) Design for $\Delta \varphi=45^{\circ} \pm 1^{\circ}$. Curve 1: $w_{1}=1.2 \mathrm{~mm}$, $w_{2}=0.85 \mathrm{~mm}, w_{3}=0.4 \mathrm{~mm}, l_{1}=14 \mathrm{~mm}, l_{2}=8.2 \mathrm{~mm}, l_{3}=9 \mathrm{~mm}$, $d=13.55 \mathrm{~mm}, H_{0}=1.2 \cdot 10^{5} \mathrm{~A} / \mathrm{m}, \epsilon_{r}=14.5-j 0.002$. Curve $2: w_{1}=1.2$ $\mathrm{mm}, l_{1}=14 \mathrm{~mm}, d=13.55 \mathrm{~mm}, H_{0}=1.2 \cdot 10^{5} \mathrm{~A} / \mathrm{m}$. (b) Design for $\Delta \varphi=90^{\circ} \pm 2^{\circ}$. Curve 1: $w_{1}=1.2 \mathrm{~mm}, w_{2}=0.85 \mathrm{~mm}, w_{3}=0.45 \mathrm{~mm}$, $l_{1}=28 \mathrm{~mm}, l_{2}=6.8 \mathrm{~mm}, l_{3}=8.5 \mathrm{~mm}, d=13.55 \mathrm{~mm}, H_{0}=1.2 \cdot 10^{5}$ $\mathrm{A} / \mathrm{m}, \epsilon_{r}=14.5-j 0.002$. Curve $2: w_{1}=1.2 \mathrm{~mm}, l_{1}=28 \mathrm{~mm}, d=13.55$ $\mathrm{mm}, H_{0}=1.2 \cdot 10^{5} \mathrm{~A} / \mathrm{m}$.

Fig. 7 shows the results of optimized structures with a two-step ferrite impedance transformer section for smallslab-width design examples. This leads to relatively long structures.

A very compact design combined with good nonreciprocal differential phase shift and low VSWR character-

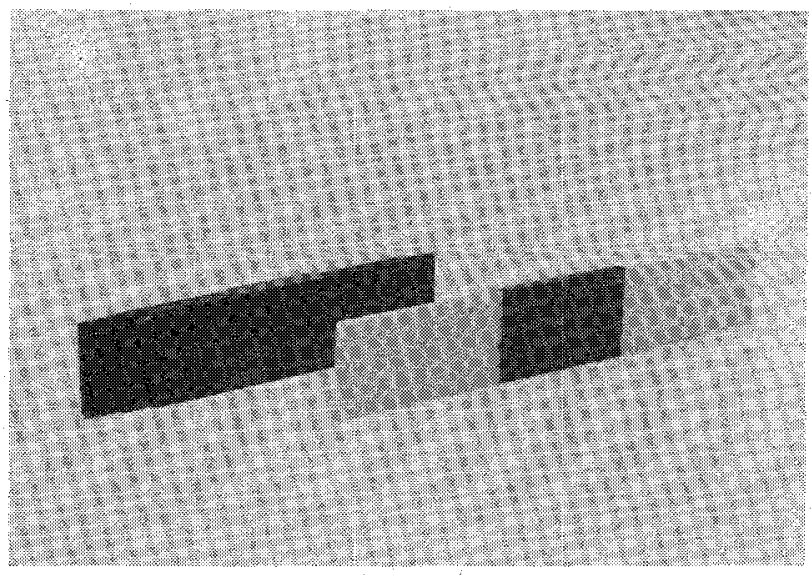

Fig. 9. Photograph of the realized prototypes: stepped ferrite structure (left) and compact ferrite nonreciprocal phase shifter with dielectric transformer sections at both ends (right), suitable for R140 waveguide housings $(15.799 \mathrm{~mm} \times 7.899 \mathrm{~mm})$. Ferrite material: TTVG-1200; dielectric material: D-15 (Trans. Tech Inc.).

istics is achieved by utilizing dielectric impedance transformer sections at both ports of a ferrite slab structure (Fig. 8). For comparison, the related curves 2 without transformer sections are included in the presentations.

In order to allow verification of the theory presented for stepped ferrite structures as well as for uniform ferrite phase shifters with dielectric transformer sections, two prototypes suitable for R140 waveguide housings and for the biasing magnet available have been fabricated (Fig. 9). The stepped-ferrite type was constructed using five commercially available TTVG- 1200 ferrite slabs with standard widths $(0.5 \mathrm{~mm})$, but of different lengths, appropriately fastened together (Fig. 9, left picture). For the hybrid type, the dielectric impedance transformer sections fastened to the uniform ferrite slab of width $1.2 \mathrm{~mm}$ were fabricated using standard D-15 material from Trans-Tech Inc., with a permittivity of 15 .

The differential phase shift and the magnitude of the input reflection coefficient of the fabricated stepped ferrite phase shifter type (Fig. 9, left) are presented in Fig. 10. Although the design is not optimum, the input reflection coefficient is better than $-20 \mathrm{~dB}$. The differential phase shift, however, is only about $10^{\circ}-15^{\circ}$. This is due to the fact that along the relatively thick middle section $(2.5 \mathrm{~mm})$, for the chosen frequency range of $16.2-16.8 \mathrm{GHz}$, three higher order modes propagate, which degrade the overall differential phase shift considerably, and to the fact that the length of the structure is limited by the biasing magnet gap. Fig. 10, therefore, may be regarded mainly from the viewpoint of verifying the theory for stepped ferrite slabs.

The compact nonreciprocal phase shifter design using a uniform ferrite slab $(1.2 \mathrm{~mm})$ together with a stepped dielectric transformer section at both ends (Fig. 9, right), however, achieves a differential phase shift of about $45^{\circ}$ (cf. Fig. 11). Compared with the optimized design of Fig. 8(a), the data $H_{0}$ and $d$ have been slightly modified in order to adapt the midband frequency range to the $\mathrm{Ku}$-band measuring equipment available. The VSWR behavior, therefore, does not correspond to that which is possible 


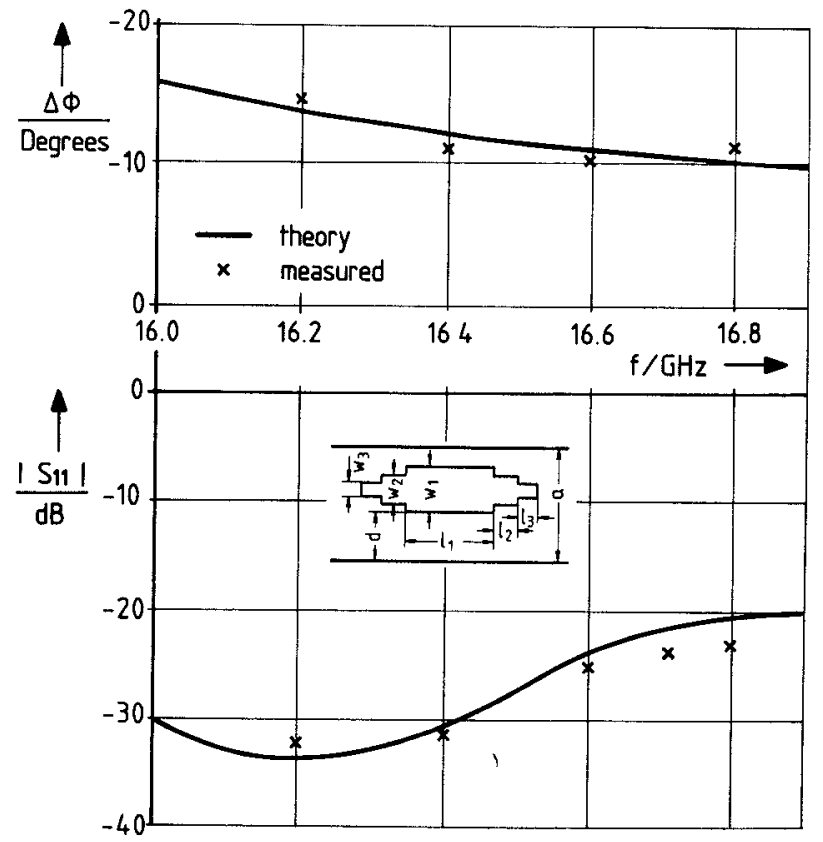

Fig. 10. Nonreciprocal differential phase shift and input reflection coefficient for the fundamental mode of the realized stepped ferrite structure (cf. Fig. 9, left). Dimensions: $a=15.799 \mathrm{~mm}, b=a / 2, w_{1}=2.5$ $\mathrm{mm}, w_{2}=1.5 \mathrm{~mm}, w_{3}=0.5 \mathrm{~mm}, l_{1}=25 \mathrm{~mm}, l_{2}=3.5 \mathrm{~mm}, l_{3}=3.5$ $\mathrm{mm}, d=12.8 \mathrm{~mm}, H_{0}=1.2 \cdot 10^{5} \mathrm{~A} / \mathrm{m}$.

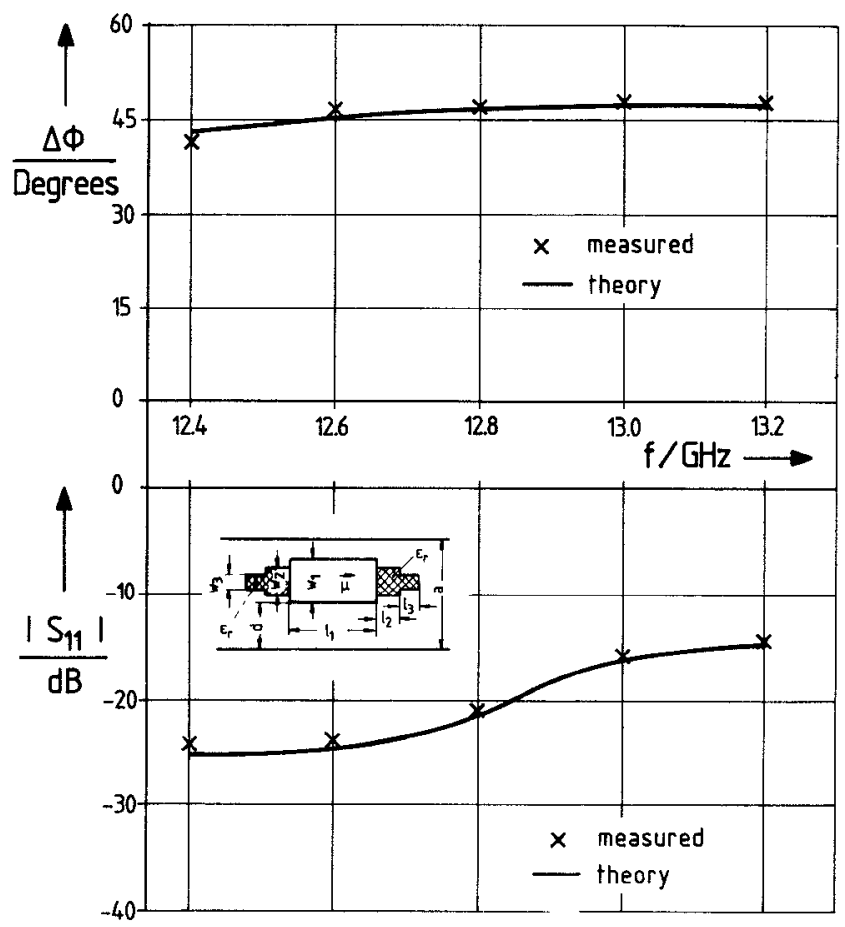

Fig. 11. Nonreciprocal differential phase shift and input reflection coefficient of the realized compact hybrid phase shifter using a uniform ferrite slab together with stepped dielectric transformer sections (cf. Fig. 9, right). Dimensions same as Fig. 8(a); exceptions: $d=13.75 \mathrm{~mm}$, $H_{0}=9 \cdot 10^{4} \mathrm{~A} / \mathrm{m}$. with such structures (cf. Fig. 8(a)). As in Fig. 10, however, the measured results agree well with the theoretically predicted values.

\section{Conclusions}

A rigorous field theory for the optimum design of ferrite-loaded waveguide nonreciprocal phase shifters is presented. Multisection ferrite or dielectric slab impedance transformers at both ends of the phase shifter achieve low VSWR values even for relatively thick ferrite middle sections. Therefore, compact phase shifter structures may be designed, as is demonstrated by numerically optimized examples for differential nonreciprocal phase shifts of $45^{\circ}$ and $90^{\circ}$. Since higher order mode interaction effects are included in the design theory, the theoretically predicted values agree well with measured results.

\section{APPENDIX}

\section{A. Scattering Coefficients in (7)}

The coefficients are

$$
\begin{aligned}
\left(S_{11}\right)^{0}= & \left(D_{E}\right)^{-1}\left(L_{E}^{(\mathrm{F})}\right) 2(M)-(U) \\
\left(S_{12}\right)^{0}= & \left(D_{E}\right)^{-1}\left(L_{E}^{(\mathrm{F})}\right)\left[2(M)\left(D_{H}\right)^{-1}\left(L_{H}^{(\mathrm{R})}\right)\right. \\
& \left.-(M)\left(\left(D_{E}\right)^{-1}\left(L_{E}^{(\mathrm{R})}\right)+\left(D_{H}\right)^{-1}\left(L_{H}^{(\mathrm{R})}\right)\right)\right] \\
& +\left(D_{E}\right)^{-1}\left(L_{E}^{(\mathrm{R})}\right) \\
\left(S_{21}\right)^{0}= & 2(M) \\
\left(S_{22}\right)^{0}= & 2(M)\left(D_{H}\right)^{-1}\left(L_{H}^{(\mathrm{R})}\right)-(M)\left[\left(D_{E}\right)^{-1}\left(L_{E}^{(\mathrm{R})}\right)\right. \\
& \left.+\left(D_{H}\right)^{-1}\left(L_{H}^{(\mathrm{R})}\right)\right] \\
(M)= & {\left[\left(D_{E}\right)^{-1}\left(L_{E}^{(\mathrm{F})}\right)+\left(D_{H}\right)^{-1}\left(L_{H}^{(\mathrm{F})}\right)\right]^{-1} } \\
(U)= & \text { unit matrix. }
\end{aligned}
$$

The diagonal matrix coefficients are

$$
D_{E m m}=\frac{a}{2} T_{m} \quad D_{H m m}=\frac{a}{2} T_{m} Y_{m}
$$

and the matrix coefficients are

$$
\begin{aligned}
L_{E m k}^{(\mathrm{F})}= & \tilde{E}_{k}^{(\mathrm{IV})(\mathrm{F})} I_{m k}^{(\mathrm{I})(\mathrm{F})}+\tilde{E}_{k}^{(\mathrm{II})(\mathrm{F})} I_{m k}^{(\mathrm{II})(\mathrm{F})} \\
& +\tilde{F}_{k}^{(\mathrm{II})(\mathrm{F})} I_{m k}^{(\mathrm{III})(\mathrm{F})}+\tilde{E}_{k}^{(\mathrm{III})(\mathrm{F})} I_{m k}^{(\mathrm{I})(\mathrm{F})} \\
L_{H m k}^{(\mathrm{F})}= & \frac{j \gamma_{k}^{(\mathrm{F})}}{\omega \mu_{0}}\left(\tilde{E}_{k}^{(\mathrm{IV})(\mathrm{F})} I_{m k}^{(\mathrm{I})(\mathrm{F})}+\tilde{E}_{k}^{(\mathrm{III})(\mathrm{F})} I_{m k}^{(\mathrm{IV})(\mathrm{F})}\right) \\
& +H_{k}^{(\mathrm{II})(\mathrm{F})} I_{m k}^{(\mathrm{II})(\mathrm{F})}+G_{k}^{(\mathrm{II})(\mathrm{F})} I_{m k}^{(\mathrm{III})(\mathrm{F})}
\end{aligned}
$$

where

$$
\begin{aligned}
H_{k}^{(\mathrm{II})(\mathrm{F})} & =\frac{j \gamma_{k}^{(\mathrm{F})} \tilde{E}_{k}^{(\mathrm{II})(\mathrm{F})}}{\omega \mu_{0} \mu_{2}}+\frac{\frac{\kappa}{\mu_{1}} k_{x k}^{(\mathrm{II})(\mathrm{F})} \tilde{F}_{k}^{(\mathrm{II})(\mathrm{F})}}{\omega \mu_{0} \mu_{2}} \\
G_{k}^{(\mathrm{II})(\mathrm{F})} & =\frac{j \gamma_{k}^{(\mathrm{F})} \tilde{F}_{k}^{(\mathrm{II})(\mathrm{F})}}{\omega \mu_{0} \mu_{2}}-\frac{\frac{\kappa}{\mu_{1}} k_{x k}^{(\mathrm{II})(\mathrm{F})} \tilde{E}_{k}^{(\mathrm{II})(\mathrm{F})}}{\omega \mu_{0} \mu_{2}} \\
\left(k_{x k}^{(\mathrm{II})(\mathrm{F})}\right)^{2} & =\left(\gamma_{k}^{(\mathrm{F})}\right)^{2}+k_{0}^{2} \cdot \epsilon_{r} \cdot \mu_{2} .
\end{aligned}
$$


The coupling integrals are given by

$$
\begin{aligned}
I_{m k}^{(\mathrm{I})(\mathrm{F})}= & \int_{x=-w-w / 2}^{-w / 2} \sin \left(k_{x k}^{(\mathrm{IV})(\mathrm{F})}(x+d+w / 2)\right) u_{m}(x) d x \\
I_{m k}^{(\mathrm{II})(\mathrm{F})}= & \int_{x=-w / 2}^{w / 2} \sin \left(k_{x k}^{(\mathrm{II})(\mathrm{F})} x\right) u_{m}(x) d x \\
I_{m k}^{(\mathrm{III})(\mathrm{F})}= & \int_{x=-w / 2}^{w / 2} \cos \left(k_{x k}^{(\mathrm{II})(\mathrm{F})} x\right) u_{m}(x) d x \\
I_{m k}^{(\mathrm{IV})(\mathrm{F})}= & \int_{x=w / 2}^{a-d-w / 2} \sin \left(k_{x k}^{(\mathrm{III})(\mathrm{F})}(a-d-w / 2-x)\right) \\
& \cdot u_{m}(x) d x
\end{aligned}
$$

with $u_{m}(x)$ given in (6), and the amplitude coefficients $E_{k}^{(\nu)}$ and $F_{k}^{(\nu)}$ of (2) in the normalized form $\tilde{E}_{k}^{(v)}, \tilde{F}_{k}^{(\nu)}$, thus the power carried by a given wave is proportional to the square of the absolute value of the amplitude coefficient [7]. The superior letter $(F)$ denotes the relations of modes directed in the forward $(+z)$ direction. Equations (A3)-(A5) hold also for the corresponding expressions denoted by $(\mathrm{R})$ for modes in the reverse direction $(-z)$ if $\gamma^{(\mathrm{F})}$ is replaced by $\gamma^{(\mathrm{R})}$; i.e., the medium is characterized by the transposed permeability tensor.

\section{B. Scattering Coefficients in (8)}

These coefficients are

$$
\begin{aligned}
\left(S_{11}\right)^{g}= & \left(S_{11}\right)^{0}+\left(S_{12}\right)^{0}(R)^{(\mathrm{R})} \\
& \cdot\left[(U)-\left(S_{11}\right)^{1}(R)^{(\mathrm{F})}\left(S_{22}\right)^{0}(R)^{(\mathrm{R})}\right]^{-1} \\
& \cdot\left(S_{11}\right)^{1}(R)^{(\mathrm{F})}\left(S_{21}\right)^{0} \\
\left(S_{12}\right)^{g}= & \left(S_{12}\right)^{0}(R)^{(\mathrm{R})} \\
& \cdot\left[(U)-\left(S_{11}\right)^{1}(R)^{(\mathrm{F})}\left(S_{22}\right)^{0}(R)^{(\mathrm{R})}\right]^{-1}\left(S_{12}\right)^{1} \\
\left(S_{21}\right)^{g}= & \left(S_{21}\right)^{1}(R)^{(\mathrm{F})} \\
& \cdot\left[(U)-\left(S_{22}\right)^{0}(R)^{(\mathrm{R})}\left(S_{11}\right)^{1}(R)^{(\mathrm{F})}\right]^{-1}\left(S_{21}\right)^{0} \\
\left(S_{22}\right)^{g}= & \left(S_{22}\right)^{1}+\left(S_{21}\right)^{1}(R)^{(\mathrm{F})} \\
& \cdot\left[(U)-\left(S_{22}\right)^{0}(R)^{(\mathrm{R})}\left(S_{11}\right)^{1}(R)^{(\mathrm{F})}\right]^{-1} \\
& \cdot\left(S_{22}\right)^{0}(R)^{(\mathrm{R})}\left(S_{12}\right)^{1} .
\end{aligned}
$$

The diagonal matrix coefficients are

$$
R_{n n}^{(\mathrm{F})}=\exp \left(-\gamma^{(\mathrm{F})} l\right) \quad R_{n n}^{(\mathrm{R})}=\exp \left(+\gamma^{(\mathrm{R})} l\right)
$$

The relation between the matrix elements, i.e.,

$$
\left(\begin{array}{ll}
\left(S_{22}\right)^{1} & \left(S_{21}\right)^{1} \\
\left(S_{12}\right)^{1} & \left(S_{11}\right)^{1}
\end{array}\right)=\left(\begin{array}{ll}
\left(S_{11}^{\prime}\right)^{0} & \left(S_{12}^{\prime}\right)^{0} \\
\left(S_{21}^{\prime}\right)^{0} & \left(S_{22}^{\prime}\right)^{0}
\end{array}\right)
$$

may be utilized for calculating the elements of $(S)^{1}$ by the corresponding elements $\left(S^{\prime}\right)^{0}$, which are given by (A1) for the transposed permeability tensor.

\section{REFERENCES}

[1] D. Polder, "On the theory of ferromagnetic resonance," Phil. Mag., vol. 40, pp. 100-115, 1949.

[2] G. T. Rado, "Theory of the microwave permeability tensor and Faraday effect in nonsaturated ferromagnetic materials," Phys. Rev., vol. 79, p. 529, 1953.

[3] B. K. J. Lax, K. J. Button, and L. M. Roth, "Ferrite phase shifters in rectangular waveguide," $J$. Appl. Phys., vol. 25, pp. 1413-1421, 1954.

[4] B. Lax and K. Button, Microwave Ferrites and Ferrimagnetics. New York: McGraw-Hill, 1962.

[5] J. J. Green and F. Sandy, "Microwave characterisation of partially magnetized ferrites," IEEE Trans. Microwave Theory Tech., vol. MTT-22, pp. 641-645, June 1974.

[6] J. J. Green and F. Sandy, "A catalog of low power loss parameters and high power thresholds for partially magnetized ferrites," IEEE Trans. Microwave Theory Tech., vol. MTT-22, pp. 645-651, June 1974.

[7] R. E. Collin; Field Theory of Guided Waves. New York: McGraw Hill, ch. 6, pp. 85, 174-179, 198-209.

[8] R. E. Collin, Foundation of Microwave Engineering. New York: McGraw Hill, 1966, ch. 6.6 .

[9] A. D. Bresler, G. H. Joshi, and N. Marcuvitz, "Orthogonality properties for modes in passive and active uniform waveguides," $J$. Appl. Phys., vol. 29, pp. 794-799, 1958.

[10] R. F. Harrington and A. I. Villeneuve, "Reciprocity relationships for gyrotropic media," IRE Trans. Microwave Theory Tech., vol. MTT-6, pp. 308-310, July 1958.

[11] A. I. Villeneuve, "Orthogonality relationships for waveguides and cavities with inhomogeneous anisotropic media," IRE Trans. Microwave Theory Tech., vol. MTT-7, pp. 441-446, Oct. 1959.

[12] W. E. Hord, F. J. Rosenbaum, and C. R. Boyd, "Theory of the suppressed rotation reciprocal ferrite phase shifter," IEEE Trans. Microwave Theory Tech., vol. MTT-16, pp. 902-910, Nov. 1968.

[13] M. C. Decreton, E. F. Loute, A. S. Vander Vorst, and F. E. Gardiol, "Computer optimization of $E$-plane resonance isolators," IEEE Trans. Microwave Theory Tech., vol. MTT-19, pp. 322-331, Mar. 1971.

[14] F. E. Gardiol and A. S. Vander Vorst, "Computer analysis of E-plane resonance isolators," IEEE Trans. Microwave Theory Tech., vol. MTT-19, pp. 315-322, Mar. 1971.

[15] F. E. Gardiol, "Anisotropic slabs in rectangular waveguides," IEEE Trans. Microwave Theory Tech., vol. MTT-18, pp. 461-467, Aug. 1970.

[16] L. Lewin, "Anomalous loss at a ferrite boundary," IEEE Trans. Microwave Theory Tech., vol. MTT-20, pp. 604-607, Sept. 1972.

[17] L. R. Whicker and D. M. Bolle, "Annotated literature survey of microwave ferrite control components and materials for 1968-1974," IEEE Trans. Microwave Theory Tech., vol. MTT-23, pp. 908-918, Nov. 1975.

[18] F. J. Bernues and D. M. Bolle, "The ferrite-loaded waveguide discontinuity problem," IEEE Trans. Microwave Theory Tech., vol. MTT-22, pp. 1187-1193, Dec. 1974.

[19] T. A. Ennegren and M. M. Z. Kharadly, "Higher order mode interaction in nonreciprocal periodic structures," IEEE Trans. Microwave Theory Tech., vol. MTT-30, pp. 809-812, May 1982.

[20] K. C. O'Brien, "Microwave properties of slabs of uniformly magnetized material filling the cross section of a rectangular waveguide operating in TE modes," IEEE Trans. Microwave Theory Tech., vol. MTT-18, pp. 377-382, July 1970.

[21] W. John, "Nichtreziproke Phasenverschiebung im teilweise mit Ferrit und Dielektrikum belasteten Rechteck-Hohlleiter," Frequenz, vol. 18 , pp. 313-325, Oct. 1964.

[22] M. S. Sodha and N. C. Srivastava, "Differential phase shift at microwave frequencies using planar ferrites," IEEE Trans. Microwave Theory Tech., vol. MTT-24, pp. 215-2l6, Apr. 1976.

[23] M. Hoffmann, "Die Streumatrix von Rechteckhohlleitungen mit magnetisierten Ferriteinsätzen endlicher Länge," Arch. Elek. Übertragung, vol. 32, pp. 62-68, Feb. 1978.

[24] H. Chaloupka, "A coupled-line model for the scattering by dielectric and ferritmagnetic obstacles in waveguides," Arch. Elek. Übertragung, vol. 34, pp. 145-151, Apr. 1980.

[25] F. E. Gardiol, Introduction to Microwaves. Dedham, MA: Artech House, 1981, ch. 6.7.

[26] C. T. M. Chang, "Partially dielectric-slab-filled waveguide phase shifter," IEEE Trans. Microwave Theory Tech., vol. MTT-22, pp. 481-485, May 1974. 
[27] F. Arndt, J. Bornemann, and R. Vahldieck, "Design of multisection impedance-matched dielectric-slab filled waveguide phase shifter," IEEE Trans. Microwave Theory Tech., vol. MTT-32, pp. 34-38, 1984.

[28] P. W. Walker, "High-power mm-wave circulators and isolators designed to operate at $44 \mathrm{GHz}$," Microwave Syst. News vol. 15, pp. 101-106, Dec. 1985.

[29] N. Okamoto, "Computer-aided design of $H$-plane waveguide junctions with full-height ferrites of arbitrary shape," IEEE Trans. Microwave Theory Tech., vol. MTT-27, pp. 315-321, Apr. 1979.

[30] A.-M. Khilla and I. Wolff, "Field theory treatment of $H$-plane waveguide junction with triangular ferrite post," IEEE Trans. Microwave Theory Tech., vol. MTT-26, pp. 279-287, 1978.

[31] S. Sensiper, "Resonance loss properties of ferrites in 9Gc region," Proc. IRE, vol. 44, pp. 1334-1342, 1956.

[32] F. Arndt, A. Frye, M. Wellnitz, and R. Wirsing, "Double dielectric-slab-filled waveguide phase shifter," IEEE Trans. Microwave Theory Tech., vol. MTT-33, pp. 373-381, May 1985.

[33] M. Hoffmann and H. Severin, "Hohlleitungsmeßverfahren zur Bestimmung der Materialdaten magnetisierter Mikrowellenferrite in Abhängigkeit von der Frequenz," Arch. Elek. Übertragung vol. 32, pp. 69-74, Feb. 1978.

[34] M. Koshiba and M. Suzuki, "Finite-element analysis of $H$-plane waveguide junction with arbitrarily shaped ferrite post," IEEE Trans. Microwave Theory Tech., vol. MTT-34, pp. 103-109, Jan. 1986.

[35] P. Hlawiczka and A. Mortis, "Gyromagnetic resonance graphical design data," Proc. Inst. Elec. Eng., vol. 110, pp. 665-670, 1963.

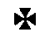

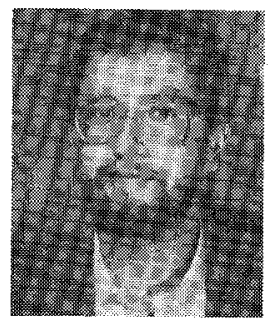

Jaroslaw Uher was born in Klomnice, Poland, on June 2, 1954. He received the M.Sc. degree in electronic engineering from the Technical University of Wroclaw, Poland, in 1978.

From 1978 to 1982 , he worked on planar ferrite engineering at the Institute of Electronic Technology, Technical University of Wroclaw. Since 1983, he has been with the Microwave Department of the University of Bremen, West Germany, where his present research activities are in the field of solving electromagnetic field problems for ferrimagnetic slab discontinuities in waveguide structures.

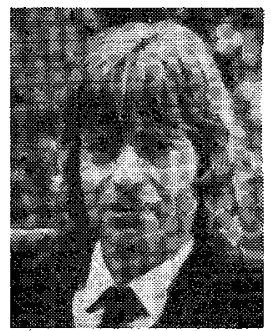

Fritz Arndt (SM'83) was born in Konstanz, Germany, on April 30, 1938. He received the Dipl. Ing., Dr. Ing., and Habilitation degrees from the Technical University of Darmstadt; West Germany, in 1963, 1968, and 1972, respectively.

From 1963 to 1973 , he worked on directional couplers and microstrip techniques at the Technical University of Darmstadt. Since 1972, he has been a Professor and Head of the Microwave Department at the University of Bremen, West Germany. His research activities are in the area of the solution of field problems of waveguide, finline, and optical waveguide structures, of antenna design, and of scattering structures.

Dr. Arndt is member of the VDE and NTG (Germany). He received the NTG award in 1970, the A. F. Bulgin Award (together with three coauthors) from the Institution of Radio and Electronic Engineers in 1983 , and the best paper award of the antenna conference JINA 1986 (France).

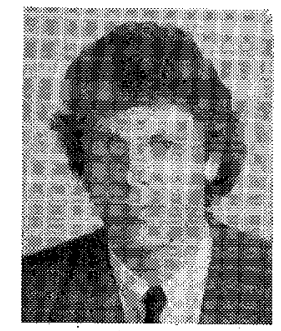

Jens Bornemann was born in Hamburg, West Germany, on May 26, 1952. He received the Dipl. Ing. and the Dr. Ing. degrees, both in electrical engineering, from the University of Bremen, Bremen, West Germany, in 1980 and 1984, respectively.

From 1980 to 1983, he was a Research Assistant at the Microwave Department of the University of Bremen, where he worked on quasiplanar waveguide configurations and computeraided $E$-plane filter design. After a two-year period as a consulting engineer, he joined the University of Bremen again, in 1985. His current research activities include millimeter-wave integrated circuit components and problems of electromagnetic field theory, especially those requiring numerical methods of solution.

Dr. Bornemann was one of the recipients of the A. F. Bulgin Premium of the Institution of Electronic and Radio Engineers (1983). 